\title{
Helium isotope systematics of volcanic gases and thermal waters of Guadeloupe Island, Lesser Antilles
}

\author{
P. Jean-Baptiste ${ }^{\mathrm{a}, *}$, P. Allard ${ }^{\mathrm{b}}$, E. Fourré ${ }^{\mathrm{a}}$, F. Parello ${ }^{\mathrm{c}}$, A. Aiuppa ${ }^{\mathrm{c}}$ \\ a Laboratoire des Sciences du Climat et de l'Environnement, CEA-Saclay, Gif-sur-Yvette, France \\ b IPGP, UMR7154 CNRS, Sorbonne Cité, Paris, France \\ c DiSTEM, Università di Palermo, Palermo, Italy
}

\section{A R T I C L E I N F O}

\section{Article history:}

Received 19 April 2014

Accepted 4 July 2014

Available online 12 July 2014

\section{Keywords:}

Volcanic gases

Helium isotope

Carbon isotope

Guadeloupe

\begin{abstract}
A B S T R A C T
The island of Guadeloupe is located in the middle of the $850 \mathrm{~km}$ long Lesser Antilles island arc. Present-day volcanic and geothermal activity is concentrated in two systems both located in the southwestern part of the island (Basse Terre): the La Soufrière volcanic complex and the Bouillante hydrothermal system, some $20 \mathrm{~km}$ to the northwest of the volcano. We report here the largest isotopic data set for helium isotopes in hydrothermal gases and waters from both systems, acquired between 1980 and $2012 .{ }^{3} \mathrm{He} /{ }^{4} \mathrm{He}$ ratios in the fumarolic gases of La Soufrière volcano have been quite homogeneous and stable over the last thirty years. The average ratio of $8.2 \pm 0.2 \mathrm{R}_{\mathrm{a}}$ confirms that the volcano is tapping a MORB-like mantle source. In contrast, the nearby Bouillante geothermal system displays a much lower ${ }^{3} \mathrm{He} /{ }^{4} \mathrm{He}$ ratio $\left(4.5 \pm 0.1 \mathrm{R}_{\mathrm{a}}\right)$. He-C elemental and isotopic relationships show that both systems are actually fed by the same magmatic source, and that their marked difference in ${ }^{3} \mathrm{He} /{ }^{4} \mathrm{He}$ results from the ${ }^{4} \mathrm{He}$ contamination of the Bouillante deep aquifer by the surrounding wallrock. This conclusion is strengthened by the spatial distribution of ${ }^{3} \mathrm{He} /{ }^{4} \mathrm{He}$ ratios which shows that La Soufrière fumaroles and the Bouillante geothermal system are the two end-members of a spatial trend of decreasing ${ }^{3} \mathrm{He} /{ }^{4} \mathrm{He}$ ratio with distance from La Soufrière summit dome, implying an increasing addition of radiogenic ${ }^{4} \mathrm{He}$ from the host rocks away from the present-day active volcanic edifice.
\end{abstract}

(C) 2014 Elsevier B.V. All rights reserved.

\section{Introduction}

Guadeloupe island belongs to the Lesser Antilles island arc, the active volcanic chain generated by the subduction of the Atlantic seafloor beneath the Caribbean plate (MacDonald et al., 2000). It is located in the middle of the arc, just south of the island of Montserrat. Present-day volcanism in Guadeloupe is active in Basse-Terre, the western part of the territory (Fig. 1). There, volcanic activity started some 3 million years ago (Briden et al., 1979; Samper et al., 2007). It migrated southward over time to give birth, some 200,000 years ago, to the Grande Découverte-La Soufrière volcanic complex (Boudon et al., 1989). The last eruption, in $1530 \mathrm{AD}$ (Boudon et al., 2008), built the $0.05 \mathrm{~km}^{3}$ andesitic lava dome of La Soufrière volcano, the highest landmark (1430 $\mathrm{m}$ asl) in Guadeloupe. Since then, intense hydrothermal activity has persisted at La Soufrière, including six phreatic eruptive phases. Volcanic gases are persistently released as fumaroles on and around the lava dome (Brombach et al., 2000; Ruzié et al., 2012) and as dissolved and/or bubbling gases in thermal springs around the volcanic edifice (Villemant et al., 2005; Ruzié et al., 2013).

\footnotetext{
* Corresponding author.

E-mail address: pjb@lsce.ipsl.fr (P. Jean-Baptiste).
}

Thermal manifestations are also widespread in the Bouillante area located on the coast some $20 \mathrm{~km}$ to the northwest of La Soufrière, including hot springs, fluids from geothermal wells, gas bubbling at sea (off the coastal area of Pointe Lézarde) and a deep submarine hot spring $(-23 \mathrm{~m})$ at the base of Ilet Pigeon (Fig. 1). The chemical composition of the thermal springs indicates a marked difference between both systems: the thermal springs of La Soufrière edifice are characteristics of young and shallow groundwaters of meteoric origin interacting with high temperature volcanic rocks and gases (Brombach et al., 2000). In contrast, Bouillante thermal waters, which have been studied intensively for their geothermal potential (Cormy et al., 1970; Demians D'Archambaud and Surcin, 1972, 1976; Sanjuan et al., 1999; Brombach et al., 2000), originate from a deeper, more mature aquifer with a marked $\mathrm{Na}-\mathrm{Cl}$ composition showing the influence of marine waters. This deep aquifer is exploited for generating electricity by a geothermal power plant located on the coast and plans for future extension are being considered.

Helium isotopes are a powerful indicator of the extent of the mantlederived contribution to crustal fluids (Ballentine et al., 2002). Their ratio varies by more than three orders of magnitude in terrestrial samples. This results from the distinct origins of ${ }^{3} \mathrm{He}$ (essentially primordial) and ${ }^{4} \mathrm{He}$ (produced by the radioactive decay of $U$ and $\mathrm{Th}$ ) and their contrasted proportions in the Earth's reservoirs. When referred to the 


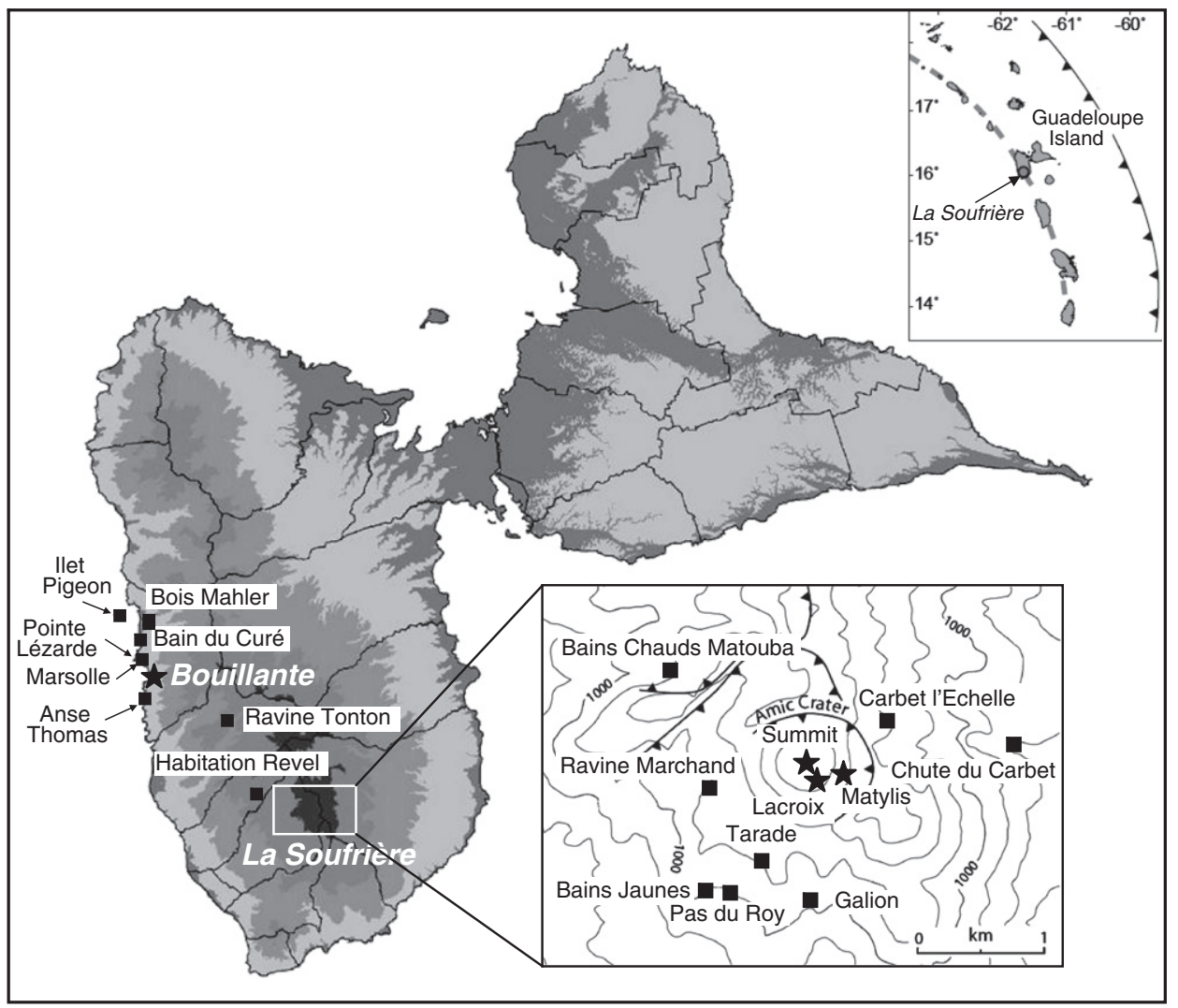

Fig. 1. Location map of the sampling points.

atmospheric ratio $\left(\mathrm{R}_{\mathrm{a}}=1.38 \times 10^{-6}\right)$, typical ${ }^{3} \mathrm{He} /{ }^{4} \mathrm{He}$ ratios vary from $<0.05 \mathrm{R}_{\mathrm{a}}$ in the continental crust to $8 \pm 1 \mathrm{R}_{\mathrm{a}}$ on average in MORB-type upper mantle, and up to $\sim 40-50 \mathrm{R}_{\mathrm{a}}$ in products of plume-related ocean islands, such as Hawaii and Iceland (Ballentine and Burnard, 2002; Graham, 2002; Stuart et al., 2003). Furthermore, helium closely follows carbon dioxide during magma degassing and the $\mathrm{CO}_{2} /{ }^{3} \mathrm{He}$ ratio of volcanic gases is one important complementary indicator of their origin (Sano and Marty, 1995; Allard et al., 1997; Marty and Tolstikhin, 1998).

Previous studies of helium isotopes in Guadeloupe (van Soest et al., 1998; Pedroni et al., 1999; Ruzié et al., 2012, 2013) have concentrated mostly on La Soufrière system and have shown that the volcano is tapping a MORB-like source with ${ }^{3} \mathrm{He} /{ }^{4} \mathrm{He}$ values around $8 \mathrm{R}$. In contrast, two results from the Bouillante geothermal power plant (van Soest et al., 1998; Pedroni et al., 1999) indicate a much lower ${ }^{3} \mathrm{He} /{ }^{4} \mathrm{He}$ value (4.5 $R_{a}$ ), raising interrogations as to whether the Bouillante hydrothermal system is fed by a magmatic source distinct from that of La Soufrière. Here, we report the results of a study conducted from 1980 to 2012, covering the two systems of La Soufrière and Bouillante and comprising a total of 92 samples from 20 sites, including both free gases (fumaroles, gas bubbles, geothermal wells) and dissolved gases in hot springs. Some of the gas samples were also analysed for their carbon isotope ratio. The aim of this study was to investigate the helium isotope composition of the main geothermal manifestations of BasseTerre (Guadeloupe), to map their spatial distribution, to study their temporal variability and to gain deeper insight into the source of the volcanic gases.

\section{Sampling and methods}

Our data set goes back to 1980 when the Lacroix fumaroles (now extinct) were sampled for the first time for helium isotopes (Allard, 1983;
Allard et al., 1983). Our first extensive survey of the helium isotopic composition of the gases and hot springs was performed in 1995. The following campaigns took place in 1996, 1999, 2006 and 2012. Water samples for helium isotopes were collected in standard refrigeration grade $3 / 8$ " copper tubes equipped with metal clamps at both ends. The tubes were flushed with the water prior to closure using either the natural water flow or a peristaltic pump connected with Tygon tubing. Fumarolic gases where collected in copper tubes or pre-evacuated glass flasked with stopcocks. When present, gas bubbles in water were also collected in the same way using an inverted funnel. Prior to 2000, He analyses were performed at LSCE-Saclay using a VG-3000 mass spectrometer (Jean-Baptiste et al., 1992) and on a MAP-215-50 mass spectrometer with simultaneous helium and neon isotope determination afterwards (Jean-Baptiste et al., 2010). Gas samples were directly connected to the high-vacuum inlet system of the mass spectrometer. Helium and neon dissolved in the water samples were first extracted under vacuum into sealed glass tubes. Typical ${ }^{4} \mathrm{He}$ and ${ }^{20} \mathrm{Ne}$ blanks are $5 \times 10^{-10}$ and $3 \times 10^{-10} \mathrm{~cm}^{3}$ STP for the mass spectrometer inlet, and $2 \times 10^{-9}$ and $1 \times 10^{-9} \mathrm{~cm}^{3}$ STP for the extraction system, respectively. For gases, analytical uncertainties on $R / R_{a}$ and helium mixing ratio are \pm 0.08 and $0.1 \mathrm{ppm}$, respectively. Analytical precision on helium and neon concentrations and on ${ }^{3} \mathrm{He} /{ }^{4} \mathrm{He}$ ratios in water samples is better than $0.8 \%$. Helium concentrations and isotopic ratios were corrected for the atmospheric air component using the measured ${ }^{20} \mathrm{Ne} /{ }^{4} \mathrm{He}$ ratio (when available) assuming a pure atmospheric origin for neon (Hilton, 1996). For the earlier samples for which no neon data were available, the helium volcanic component dissolved in water was obtained by subtracting the atmospheric air component at the solubility equilibrium (Weiss, 1971; Jean-Baptiste et al., 2009), and, for gas samples, from the measured co-amount of uncondensable gases assumed to be entirely atmospheric in origin. Comparison of the results given by both methods for waters (Table 2) shows that using the solubility 
data to derive the atmospheric component gives acceptable results as long as the helium excess in the sample is large enough (in practice, greater than $50 \%$ ).

$\mathrm{CO}_{2}$ concentration in volcanic gases was determined at Palermo University using a Perkin Elmer AutoSystem XLClarus 500 gas chromatograph equipped with a thermal conductivity detector (HWD). $\delta^{13} \mathrm{C}$ measurements were also performed at Palermo University using a Finnigan Delta Plus mass spectrometer, with an accuracy of $\pm 0.1 \%$.

\section{Results}

\subsection{Free gases}

Gas results are given in Table 1 . Volcanic gases were repeatedly sampled from the South Crater, the most active fumarolic vent at the top of La Soufrière lava dome, and from the Matylis fumarolic field extending at the base of the dome on a major volcano-tectonic fault (Ty Fault) cutting the southeast flank of the volcanic edifice. Gases were also sampled at Bains Chauds Matouba, a bubbling hot spring located on a former crater rim some $1.5 \mathrm{~km}$ to the northwest of the dome (Fig. 1). Gases representative of the Bouillante geothermal system were sampled at the well head of the geothermal power plant and off the shoreline of Pointe Lézarde (Fig. 1): in this latter area, degassing occurs over a wide area, with gas bubbles rising intermittently and randomly from the seafloor. These bubbles were collected on their way to the surface by scuba diving.

\subsubsection{La Soufrière system}

The air-corrected ${ }^{3} \mathrm{He} /{ }^{4} \mathrm{He}$ values for La Soufrière gas samples are quite homogeneous and quite stable over time (Fig. 2). The average value of $8.2 \pm 0.2 R_{a}$ is in good agreement with the results already published by van Soest et al., 1998 (7.94-7.96 R $\mathrm{a}_{\mathrm{a}}$; Pedroni et al., 1999

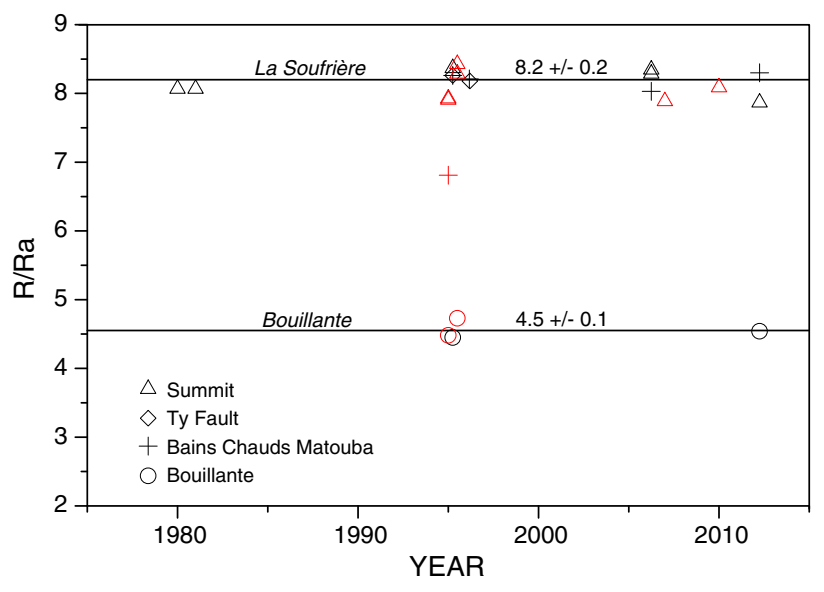

Fig. 2. ${ }^{3} \mathrm{He} /{ }^{4} \mathrm{He}$ time-series in the volcanic gases (in red: data from Van Soest et al., 1998; Pedroni et al., 1999; Ruzié et al., 2012; in black: this work).

(8.32-8.46 $\mathrm{R}_{\mathrm{a}}$ ) and Ruzié et al., $2012\left(7.9-8.1 \mathrm{R}_{\mathrm{a}}\right)$ and evidences a MORB-type mantle source feeding the volcanic system. As classically observed in arc-related fluids (Fischer and Marty, 2005), the mean value of $\left(\mathrm{CO}_{2} /{ }^{3} \mathrm{He}\right)$ ratios, $(11.2 \pm 2.7) \times 10^{9}$, is $\sim 5$ times higher than the MORB value of $(2 \pm 1) \times 10^{9}$ (Marty and Jambon, 1987; Marty and Tolstikhin, 1998), due to the addition of slab-derived carbon supplied by sedimentary marine carbonate and organic matter. The mean $\delta^{13} \mathrm{C}$ value of the volcanic $\mathrm{CO}_{2},(-3.2 \pm 0.3) \%$, is also significantly higher than the MORB value of $(-6.5 \pm 2) \%$ (Javoy et al., 1986; Marty and Jambon, 1987; Sano and Marty, 1995), indicating that the carbonate carbon component $\left(\delta^{13} \mathrm{C} \sim 0 \%\right.$ oo is predominant over the organic carbon component $\left(\delta^{13} \mathrm{C} \sim-30 \pm 10 \%\right.$ o-Hoefs, 1980 ; Sano and

Table 1

Helium and carbon isotopic compositions of the volcanic gases of Guadeloupe. Words and numbers in italic refer to literature data.

\begin{tabular}{|c|c|c|c|c|c|c|c|c|c|c|}
\hline \multirow[t]{2}{*}{ Site } & \multirow[t]{2}{*}{ Date } & \multirow[t]{2}{*}{$\mathrm{T}^{\circ} \mathrm{C}^{\circ}$} & \multirow{2}{*}{$\frac{\mathrm{CO}_{2}}{\mathrm{~mol} \%}$} & \multirow[t]{2}{*}{${ }^{4} \mathrm{He} /{ }^{20} \mathrm{Ne}$} & \multirow{2}{*}{$\frac{(\mathrm{He}) \mathrm{c}}{\mathrm{ppmv}}$} & \multirow{2}{*}{$\frac{{ }^{3} \mathrm{He} /{ }^{4} \mathrm{He}}{(\mathrm{R} / \mathrm{Ra})_{\mathrm{m}}}$} & \multirow{2}{*}{$\frac{{ }^{3} \mathrm{He} /{ }^{4} \mathrm{He}}{(\mathrm{R} / \mathrm{Ra})_{\mathrm{c}}}$} & \multirow{2}{*}{$\frac{\mathrm{CO}_{2} /\left({ }^{3} \mathrm{He}\right) \mathrm{C}}{\left(\times 10^{9}\right)}$} & \multirow{2}{*}{$\frac{\delta^{13} \mathrm{C}}{\%}$} & \multirow[t]{2}{*}{ References } \\
\hline & & & & & & & & & & \\
\hline South Crater (fum-AN9) & 03/1995 & 98.0 & 90.6 & & 6 & 8.23 & 8.4 & 13.3 & -3.2 & This work \\
\hline South Crater (fum-AN2) & 03/1995 & 97.7 & & & & 7.63 & 7.83 & & -3.2 & This work \\
\hline South Crater & 1995 & 94 & & & & 7.93 & 7.94 & 11.5 & -3.2 & Van Soest et al., 1998 \\
\hline South Crater & 1995 & 94 & & & & 7.91 & 7.96 & 11.7 & -3.1 & Van Soest et al., 1998 \\
\hline South Crater & 06/1995 & 108 & 99 & & 4.95 & 8.31 & 8.32 & 17.4 & -3.01 & Pedroni et al., 1999 \\
\hline South Crater & 06/1995 & 108 & 65 & & 6.35 & 8.46 & 8.46 & 8.8 & -3.02 & Pedroni et al., 1999 \\
\hline South Crater (fum-AN2) & 02/1996 & 97.3 & 92.2 & & & 7.17 & & & -3.2 & This work \\
\hline South Crater (fum-AN2) & 01/1999 & 98.9 & 92.1 & & 9.7 & & & & & This work \\
\hline South Crater (fum-AN2) & $03 / 2006$ & 108.5 & 66.9 & 10.7 & 5.3 & 8.16 & 8.38 & 11.2 & & This work \\
\hline South Crater (fum-AN2) & $03 / 2006$ & 108.5 & 66.6 & 38.9 & 5.4 & 8.26 & 8.32 & 10.8 & & This work \\
\hline South Crater & 2007 & & & & 5.32 & & 7.8 & & & Ruzié et al., 2012 \\
\hline South Crater & 2010 & & & & 6.78 & & 7.6 & & & Ruzié et al., 2012 \\
\hline South Crater & $03 / 2012$ & 107.6 & 67.3 & 18.3 & 6.2 & 7.78 & 7.90 & 10.1 & & This work \\
\hline Lacroix fumarole & $01 / 1980$ & 103.8 & 80.0 & & 8.3 & 8.06 & 8.10 & 8.7 & -3.7 & This work \\
\hline Lacroix fumarole & 04/1981 & 97.8 & 73.8 & & 7.7 & 8.10 & 8.10 & 8.6 & -3.9 & This work \\
\hline Ty Fault (Matylis) & 03/1995 & 97.5 & 89.6 & & & 7.98 & 8.26 & & -2.8 & This work \\
\hline Ty Fault (Matylis) & 02/1996 & 96.4 & & & & 8.18 & 8.18 & & -3.2 & This work \\
\hline Ty Fault (Matylis) & 02/1996 & & & & & 7.86 & & & & This work \\
\hline Ty Fault (Matylis) & 01/1999 & 96.6 & 88.3 & & 6.7 & & & & & This work \\
\hline Bain Chaud Matouba & 1985 & & 5.13 & & & & & & & Fabriol and Ouzounian, 1985 \\
\hline Bain Chaud Matouba & 03/1995 & 59.9 & 1.04 & & 10.5 & 4.56 & 8.26 & 0.16 & -6.0 & This work \\
\hline Bain Chaud Matouba & 1995 & 55.5 & & & & 3.27 & 6.81 & & & Van Soest et al., 1998 \\
\hline Bain Chaud Matouba & 02/1996 & 58.6 & 7.9 & & & 4.47 & & & -11.3 & This work \\
\hline Bain Chaud Matouba & 01/1999 & 58.6 & & & & & & & & This work \\
\hline Bain Chaud Matouba & $03 / 2006$ & 58.5 & 3.87 & 0.67 & 6.1 & 4.69 & 8.03 & 0.98 & & This work \\
\hline Bain Chaud Matouba & $03 / 2012$ & 58.5 & & 0.60 & 8.5 & 4.43 & 8.30 & & & This work \\
\hline Bouillante $\mathrm{BO} 2$ & 1991 & & 93.6 & & 40 & & & & -2.6 & Abou Akar et al., 1992 \\
\hline Bouillante (Pte Lézarde) & 03/1995 & & & & 494 & 4.41 & 4.45 & & & This work \\
\hline Bouillante BO4 & 03/1995 & & & & & & & & -3.4 & This work \\
\hline Bouillante & 1995 & 55.5 & & & & 4.46 & 4.48 & & -2.9 & Van Soest et al., 1998 \\
\hline Bouillante & 06/1995 & & 38 & & 203 & 4.72 & 4.73 & 0.29 & -2.43 & Pedroni et al., 1999 \\
\hline Bouillante BO4 & 01/1999 & & 90.4 & & & & & & & This work \\
\hline Bouillante BO6 & $11 / 2012$ & & 94.3 & 31.87 & 26.2 & 4.50 & 4.53 & 5.80 & & This work \\
\hline
\end{tabular}


Marty, 1995). The gases from the Bains Chauds Matouba hot spring were not taken into account in the calculation of the $\left(\mathrm{CO}_{2} /{ }^{3} \mathrm{He}\right)$ and $\delta^{13} \mathrm{C}$ mean values because of their peculiar composition (low $\mathrm{CO}_{2}$ and very high $\mathrm{N}_{2}$ concentrations) which indicates a strong $\mathrm{CO}_{2}$ elemental and isotopic fractionation during previous gas-water separation (Ruzié et al., 2013).

Table 2

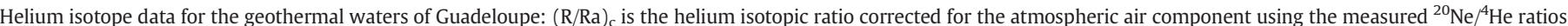

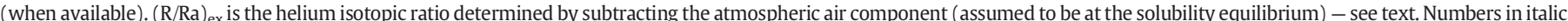
refer to literature data.

\begin{tabular}{|c|c|c|c|c|c|c|c|c|c|c|}
\hline \multirow[t]{2}{*}{ Site } & \multirow[t]{2}{*}{ Date } & \multirow{2}{*}{$\frac{\text { Temperature }}{\left({ }^{\circ} \mathrm{C}\right)}$} & \multirow{2}{*}{$\frac{\text { Conductivity }}{\left(\mu \mathrm{S}-25^{\circ} \mathrm{C}\right)}$} & \multirow[t]{2}{*}{$\mathrm{pH}$} & \multirow{2}{*}{$\frac{\left[{ }^{4} \mathrm{He}\right]}{\times \mathrm{E}+8 \mathrm{~cm}^{3} / \mathrm{g}}$} & \multirow{2}{*}{$\frac{\left[{ }^{3} \mathrm{He}\right]}{\times \mathrm{E}+14 \mathrm{~cm}^{3} / \mathrm{g}}$} & \multirow{2}{*}{$\frac{\left.{ }^{[20} \mathrm{Ne}\right]}{\times \mathrm{E}+8 \mathrm{~cm}^{3} / \mathrm{g}}$} & \multirow[t]{2}{*}{$(\mathrm{R} / \mathrm{Ra})_{\text {meas }}$} & $(\mathrm{R} / \mathrm{Ra})_{\mathrm{ex}}$ & $(\mathrm{R} / \mathrm{Ra})_{\mathrm{c}}$ \\
\hline & & & & & & & & & & \\
\hline Carbet l'Echelle & 03/1995 & 23.0 & 1410 & 5.51 & 28.92 & 268.19 & & 6.72 & 7.8 & \\
\hline Carbet l'Echelle & 01/1996 & 23.2 & & & 27.20 & 233.85 & & 6.23 & 7.2 & \\
\hline Carbet l'Echelle & 01/1999 & 22.4 & 1920 & 5.44 & 25.68 & 246.30 & & 6.95 & 8.2 & \\
\hline Carbet l'Echelle & 03/2006 & 21.2 & 936 & 5.19 & 18.3 & 159.10 & 14.5 & 6.30 & 8.0 & 7.76 \\
\hline Galion & 03/1995 & 42.5 & 1200 & 4.80 & 8.55 & 58.52 & & 4.96 & 8.7 & \\
\hline Galion & 01/1996 & 42.9 & & & 5.82 & 38.15 & & 4.75 & & \\
\hline Galion & 01/1999 & 41.9 & 1557 & 4.95 & 5.81 & 42.98 & & 5.36 & & \\
\hline Galion & $03 / 2006$ & 44.2 & 1890 & 4.93 & 5.29 & 35.99 & 7.2 & 4.93 & & 7.55 \\
\hline Galion-1 & $03 / 2012$ & 47.9 & 2047 & 4.29 & 5.49 & 44.46 & 4.78 & 5.87 & & 7.57 \\
\hline Galion-2 & $03 / 2012$ & 47.9 & 2047 & 4.29 & 5.20 & 41.67 & 4.62 & 5.81 & & 7.54 \\
\hline Ravine Marchand & $03 / 2006$ & 45.3 & 1220 & 5.30 & 13.6 & 120.30 & 9.6 & 6.41 & 8.8 & 7.81 \\
\hline Ravine Marchand-1 & $03 / 2012$ & 44.6 & 1105 & 5.29 & 23.0 & 233.86 & 6.08 & 7.37 & 8.8 & 7.91 \\
\hline Ravine Marchand-2 & $03 / 2012$ & 44.6 & 1105 & 5.29 & 22.9 & 232.21 & 5.99 & 7.35 & 8.7 & 7.88 \\
\hline Tarade & 03/1995 & 34.7 & 1185 & 6.02 & 6.62 & 21.74 & & 2.38 & & \\
\hline Tarade & 01/1996 & 33.1 & & & 6.45 & 26.08 & & 2.93 & 6.8 & \\
\hline Tarade & 01/1999 & 33.2 & 1492 & 5.98 & 4.98 & 16.08 & & 2.34 & & \\
\hline Tarade & $03 / 2006$ & 36.8 & 1540 & 5.87 & 4.93 & 16.53 & 13.4 & 2.43 & & 7.49 \\
\hline Tarade-1 & $03 / 2012$ & 41.5 & 1645 & 5.97 & 5.52 & 23.73 & 12.4 & 3.12 & & 7.13 \\
\hline Tarade-2 & $03 / 2012$ & 41.5 & 1645 & 5.97 & 5.51 & 24.01 & 12.2 & 3.16 & & 7.10 \\
\hline Tarade-3 & $03 / 2012$ & 41.6 & 1534 & 5.97 & 6.04 & 30.29 & 11.8 & 3.63 & & 7.15 \\
\hline Tarade-4 & $03 / 2012$ & 41.6 & 1534 & 5.97 & 6.28 & 33.37 & 11.6 & 3.85 & & 7.15 \\
\hline Pas du Roy & 03/1995 & 31.8 & 1023 & 5.65 & & & & & & \\
\hline Pas du Roy & $03 / 2006$ & 33.5 & 990 & 5.46 & 5.74 & 22.89 & 14.5 & 2.89 & & 7.66 \\
\hline Pas du Roy-1 & $03 / 2012$ & 34.6 & 1018 & 5.59 & 5.43 & 19.18 & 14.1 & 2.56 & & 6.98 \\
\hline Pas du Roy-2 & $03 / 2012$ & 34.6 & 1018 & 5.59 & 5.25 & 17.53 & 14.0 & 2.42 & & 6.90 \\
\hline Bains Jaunes (main) & 03/1995 & 28.9 & 745 & 5.09 & 5.58 & 11.47 & & 1.49 & & \\
\hline Bains Jaunes (main) & 01/1999 & 27.9 & 789 & 5.15 & 4.24 & 9.01 & & 1.54 & & \\
\hline Bains Jaunes (main) & $03 / 2006$ & 29.8 & 720 & 5.26 & 4.03 & 6.06 & 14.4 & 1.09 & & 6.59 \\
\hline Bains Jaunes (main) & $03 / 2012$ & 35.3 & 780 & 5.35 & 4.01 & 6.26 & 13.7 & 1.13 & & 7.19 \\
\hline Bains Jaunes (side)-1 & $03 / 2012$ & 29.4 & 725 & & 4.43 & 8.47 & 14.6 & 1.39 & & 6.11 \\
\hline Bains Jaunes (side)-2 & $03 / 2012$ & 29.4 & 725 & & 4.51 & 8.64 & 14.9 & 1.39 & & 6.08 \\
\hline Bains Chauds Matouba & 03/1995 & 59.9 & 649 & 5.57 & 6.56 & 31.23 & & 3.45 & 6.8 & \\
\hline Bains Chauds Matouba & 01/1996 & 58.6 & & & 7.65 & 47.19 & & 4.47 & 7.9 & \\
\hline Bains Chauds Matouba & 01/1999 & 58.6 & 1197 & 5.93 & 5.12 & 20.28 & & 2.87 & & \\
\hline Bains Chauds Matouba & $03 / 2006$ & 58.5 & 1696 & 5.50 & 7.11 & 40.03 & 13.8 & 4.08 & 7.7 & 8.67 \\
\hline Bains Chauds Matouba-1 & $03 / 2012$ & 58.5 & 1014 & 5.50 & 6.90 & 38.38 & 13.5 & 4.03 & 7.8 & 8.58 \\
\hline Bains Chauds Matouba-2 & $03 / 2012$ & 58.5 & 1014 & 5.50 & 5.95 & 28.98 & 13.2 & 3.53 & 8.1 & 8.99 \\
\hline Chute du Carbet & 03/1995 & 45.7 & 0.623 & 6.63 & 14.70 & 97.17 & & 4.79 & 6.3 & \\
\hline Chute du Carbet & 01/1996 & 45.6 & & & 20.10 & 127.87 & & 4.61 & 5.5 & \\
\hline Chute du Carbet & 01/1999 & 45.5 & 1700 & 6.62 & 8.50 & 45.75 & & 3.90 & 6.6 & \\
\hline Chute du Carbet & $03 / 2006$ & 45.0 & 1414 & 6.34 & 15.4 & 106.90 & 13.2 & 5.03 & 6.5 & 6.39 \\
\hline Chute du Carbet- 1 & $03 / 2012$ & 43.8 & 909 & & 16.2 & 114.64 & 14.4 & 5.13 & 6.6 & 6.59 \\
\hline Chute du Carbet-2 & $03 / 2012$ & 42.5 & 875 & & 14.6 & 99.79 & 14.0 & 4.95 & 6.5 & 6.49 \\
\hline Chute du Carbet-3 & $03 / 2012$ & 41.8 & 806 & & 8.43 & 45.85 & 13.7 & 3.94 & 6.8 & 6.59 \\
\hline Chute du Carbet- 4 & $03 / 2012$ & 41.8 & 806 & & 9.61 & 55.04 & 14.3 & 4.15 & 6.6 & 6.57 \\
\hline Habitation Revel & $03 / 1995$ & 33.6 & 180 & 6.66 & 4.40 & 20.28 & & 3.34 & & \\
\hline Habitation Revel & 01/1999 & 31.4 & 260 & 6.72 & 7.76 & 30.52 & & 2.85 & 5.2 & \\
\hline Habitation Revel & $03 / 2006$ & 25.2 & 298 & 6.43 & 11.9 & 67.33 & 15.4 & 4.10 & 5.9 & 5.81 \\
\hline Habitation Revel-1 & $03 / 2012$ & 32.7 & 284 & 6.53 & 15.9 & 97.88 & 15.0 & 4.46 & 5.8 & 5.72 \\
\hline Habitation Revel-2 & $03 / 2012$ & 32.7 & 284 & 6.53 & 13.8 & 81.11 & 15.3 & 4.26 & 5.7 & 5.75 \\
\hline Ravine Tonton & 03/1995 & 39.2 & 125 & 7.60 & 23.06 & 135.88 & & 4.27 & 5.0 & \\
\hline Anse Thomas & 03/1995 & 85.3 & 18,400 & 7.55 & 9.64 & 38.98 & & 2.93 & 3.5 & \\
\hline Anse Thomas-1 & $03 / 2012$ & 72.5 & 19,300 & 6.80 & 28.1 & 163.99 & 10.9 & 4.23 & 4.6 & 4.72 \\
\hline Anse Thomas-2 & $03 / 2012$ & 72.5 & 19,300 & 6.80 & 25.5 & 145.33 & 11.5 & 4.13 & 4.6 & 4.69 \\
\hline Bouillante BO4 & 03/1995 & 33.5 & 19,600 & & 439.20 & 2685.01 & & 4.43 & 4.46 & \\
\hline Bain du Curé & 03/1995 & 40.3 & 223 & 7.13 & 7.26 & 21.84 & & 2.18 & 3.8 & \\
\hline Bain du Curé & 01/1999 & 39.6 & 223 & & 6.03 & 15.39 & & 1.85 & & \\
\hline Bain du Curé-1 & $03 / 2012$ & 40.7 & 319 & & 6.90 & 21.84 & 15.2 & 2.29 & 4.3 & 4.59 \\
\hline Bain du Curé-2 & $03 / 2012$ & 40.7 & 319 & & 6.96 & 21.59 & 15.7 & 2.25 & 4.2 & 4.64 \\
\hline Marsolle & $03 / 1995$ & 46.3 & 528 & 7.03 & 10.30 & 41.50 & & 2.92 & 4.2 & \\
\hline Marsolle-1 & $03 / 2012$ & 47.2 & 839 & 7.05 & 11.9 & 53.45 & 15.3 & 3.26 & 4.4 & 4.66 \\
\hline Marsolle-2 & $03 / 2012$ & 47.2 & 839 & 7.05 & 11.2 & 48.92 & 15.1 & 3.17 & 4.4 & 4.61 \\
\hline Bois Mahler & 03/1995 & 36.3 & 227 & 7.25 & 8.05 & 25.44 & & 2.29 & 3.8 & \\
\hline Bois Mahler & 01/1999 & 33.8 & 227 & & 6.70 & 20.99 & & 2.27 & 4.6 & \\
\hline Bois Mahler-1 & $03 / 2012$ & 36.2 & 310 & & 8.07 & 29.51 & 17.2 & 2.65 & 4.5 & 5.23 \\
\hline Bois Mahler-2 & $03 / 2012$ & 36.2 & 310 & & 8.27 & 29.99 & 17.4 & 2.63 & 4.4 & 5.11 \\
\hline Ilet Pigeon & 03/1995 & 49.5 & 28,500 & 7.88 & 7300.00 & $33,143.46$ & & 3.29 & 3.29 & \\
\hline
\end{tabular}




\subsubsection{Bouillante system}

Helium isotope composition of the gases from the Bouillante geothermal area is also stable over time (Fig. 2). Although their carbon isotopic composition differs only slightly from that of La Soufrière gases, with a mean $\delta{ }^{13} \mathrm{C}$ value of $(-3.0 \pm 0.4) \%$, their ${ }^{3} \mathrm{He} /{ }^{4} \mathrm{He}$ ratio $\left(4.5 \pm 0.1 R_{a}\right)$ is much lower. This strong helium isotope contrast between both systems thus raises the question of the possibility of a different magmatic source feeding the geothermal reservoir (see discussion $§ 4)$.

\subsection{Thermal springs}

Thermal spring results are given in Table 2. Their location is shown in Fig. 1. Most groundwaters in La Soufrière area have transit times of a few months only (Pascaline et al., 1982; Bigot et al., 1994). Because of this low residence time and of the low tritium (Pascaline et al., 1982) and high helium contents of the waters, the tritiugenic ${ }^{3} \mathrm{He}$ contribution to the measured ${ }^{3} \mathrm{He} /{ }^{4} \mathrm{He}$ ratio is negligible. Our results are in excellent agreement with those published by Ruzié et al., 2013. Helium isotope data are plotted in Fig. 3. Thermal springs in the close vicinity of the volcano plot along a line the slope of which defines a ${ }^{3} \mathrm{He} /{ }^{4} \mathrm{He}$ ratio which is in close agreement with that of the summit fumaroles. More distant springs are characterized by lower ${ }^{3} \mathrm{He} /{ }^{4} \mathrm{He}$ values, thus displaying a decreasing trend with distance from the volcanic axis. Helium isotope results for the thermal springs in the Bouillante area indicate a ${ }^{3} \mathrm{He} /{ }^{4} \mathrm{He}$ ratio which is fully consistent with that of the free gases sampled at the geothermal station and in the marine bubbles off Pointe Lézarde. The lowest ${ }^{3} \mathrm{He} /{ }^{4} \mathrm{He}$ of the whole data set $\left(3.3 \mathrm{R}_{\mathrm{a}}\right.$ ), coinciding with the highest helium concentration $\left(7.3 \times 10^{-5} \mathrm{~cm}^{3} \mathrm{STP} / \mathrm{g}\right)$, is found in the small submarine hot spring located at $-23 \mathrm{~m}$ off Ilet Pigeon, a tiny island located a few kilometres to the NE of the Bouillante power station. It also corresponds to the sampling point which is the most distant from La Soufrière volcanic centre.

\section{Discussion}

${ }^{3} \mathrm{He} /{ }^{4} \mathrm{He}$ ratios for La Soufrière clearly fall in the MORB range and thus indicate a MORB-type mantle source feeding the volcanic system.

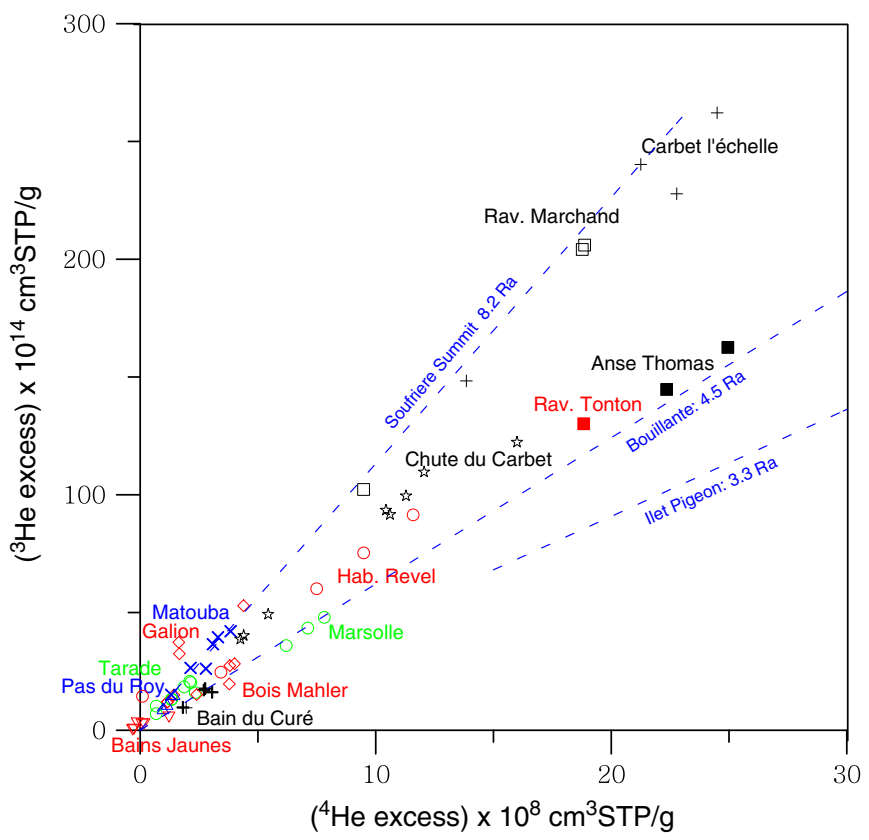

Fig. 3. Relationship between the non-atmospheric ${ }^{3} \mathrm{He}$ and ${ }^{4} \mathrm{He}$ concentrations in hot spring waters. The non-atmospheric component is obtained by subtracting the dissolved air component (calculated using the neon concentration or the solubility value when no neon is available - see text) from the measured concentrations.

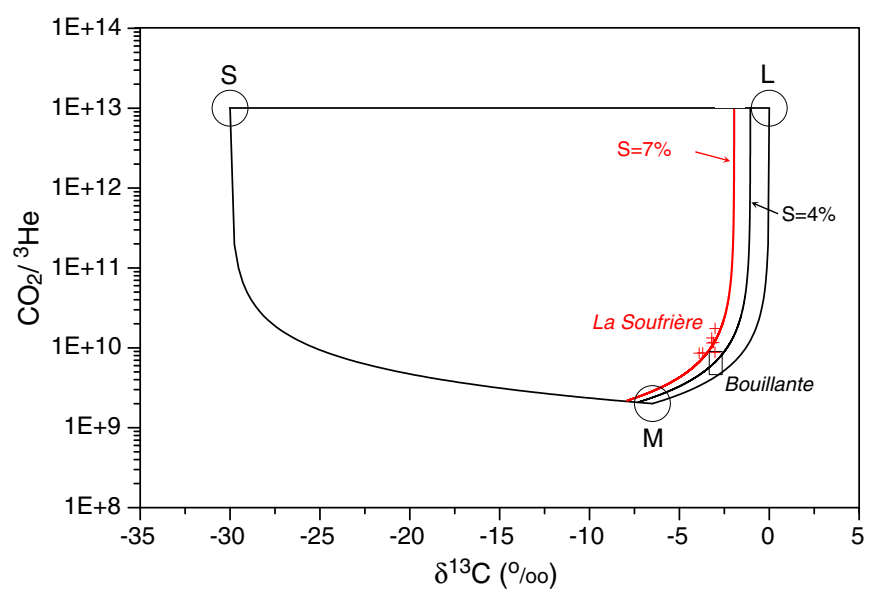

Fig. 4. $\left(\mathrm{CO}_{2} /{ }^{3} \mathrm{He}\right)$ vs $\delta^{13} \mathrm{C}$ diagram for summit fumaroles and Bouillante gases in relation to the three major end-members contributing to $\mathrm{He}$ and $\mathrm{CO}_{2}$ in geothermal fluids ( $\mathrm{M}$ represents the upper mantle end-member, $\mathrm{L}$ and $\mathrm{O}$ represent the marine carbonate-limestone and organic material, respectively). The black rectangle represents the mean of the $\left(\mathrm{CO}_{2} /{ }^{3} \mathrm{He}\right)$ and $\delta^{13} \mathrm{C}$ values for the Bouillante system deduced from our $\mathrm{CO}_{2}$, helium and $\delta^{13} \mathrm{C}$ data (see Table 1 ).

Fig. 4 displays the $\left(\mathrm{CO}_{2} /{ }^{3} \mathrm{He}\right)$ vs $\delta^{13} \mathrm{C}$ data for the summit fumaroles of La Soufrière and for the Bouillante gases in relation to the three major end-members contributing to $\mathrm{He}$ and $\mathrm{CO}_{2}$ in volcanic and geothermal fluids (Sano and Marty, 1995): M represents the upper mantle endmember with $\left(\mathrm{CO}_{2} /{ }^{3} \mathrm{He}\right)_{\mathrm{M}}=(2 \pm 1) \times 10^{9}$ (Marty and Jambon, 1987) and $\delta^{13} \mathrm{C}_{\mathrm{M}}=(-6.5 \pm 2) \%$ (Javoy et al., 1986; Marty and Jambon, 1987; Sano and Marty, 1995); L represents the sedimentary carbonate-limestone end-member with $\left(\mathrm{CO}_{2} /{ }^{3} \mathrm{He}\right)_{\mathrm{L}} \approx 1 \times 10^{13}$ (Sano and Marty, 1995; Kulongoski et al., 2013) and $\delta^{13} \mathrm{C}_{\mathrm{L}}=0 \%$; and $\mathrm{S}$ the sedimentary organic end-member with $\left(\mathrm{CO}_{2} /{ }^{3} \mathrm{He}\right)_{\mathrm{S}} \approx 1 \times 10^{13}$ (Sano and Marty, 1995; Kulongoski et al., 2013) and $\delta^{13} \mathrm{C}_{\mathrm{S}} \approx(-30 \pm$ $10 \%$ (Hoefs, 1980; Sano and Marty, 1995). The $\left(\mathrm{CO}_{2} /{ }^{3} \mathrm{He}\right)$ value of most crustal $\mathrm{CO}_{2}$-rich continental gases is in the range $10^{12}-10^{14}$ (O'Nions and Oxburgh, 1988). Therefore, the adopted value of $10^{13}$ for the $\mathrm{L}$ and $\mathrm{S}$ end-members is not well constrained. However, as already noted by Sano and Marty (1995), assuming either $10^{12}$ or $10^{14}$ does not significantly change the proportion of $\mathrm{CO}_{2}$ derived from each of the three end-members (Fig. 4).

The respective proportions $\mathrm{M}, \mathrm{L}$ and $\mathrm{S}$ can be calculated from the following set of equations:

$$
\begin{aligned}
& \left(\delta^{13} \mathrm{C}\right)=\mathrm{M}\left(\delta^{13} \mathrm{C}\right)_{\mathrm{M}}+\mathrm{L}\left(\delta^{13} \mathrm{C}\right)_{\mathrm{L}}+\mathrm{S}\left(\delta^{13} \mathrm{C}\right)_{\mathrm{S}} \\
& \left({ }^{3} \mathrm{He} / \mathrm{CO}_{2}\right)=\mathrm{M}\left({ }^{3} \mathrm{He} / \mathrm{CO}_{2}\right)_{\mathrm{M}}+\mathrm{L}\left({ }^{3} \mathrm{He} / \mathrm{CO}_{2} /\right)_{\mathrm{L}}+\mathrm{S}\left({ }^{3} \mathrm{He} / \mathrm{CO}_{2} /\right)_{\mathrm{S}}
\end{aligned}
$$

$\mathrm{L}+\mathrm{M}+\mathrm{S}=1$

For La Soufrière, the calculated mixing coefficients are $M=18 \pm 1 \%$, $\mathrm{L}=75 \pm 5 \%$ and $\mathrm{S}=7 \pm 4 \%$, in agreement with typical values for volcanic arcs (Oppenheimer et al., 2013). For the Bouillante area, the mixing proportions are $30 \pm 1 \%, 66 \pm 4 \%$ and $4 \pm 3 \%$, respectively. The difference with La Soufrière system is almost entirely due to the lower $\left(\mathrm{CO}_{2} /{ }^{3} \mathrm{He}\right)$ ratio $\left(5.8 \times 10^{9}\right)$. The validity of the $\left(\mathrm{CO}_{2} /{ }^{3} \mathrm{He}\right)-\delta^{13} \mathrm{C}$ mixing diagram is based on the assumption that $\mathrm{CO}_{2}$ and He are not fractionated during their transport to the Earth's surface. However, $\mathrm{CO}_{2}$ and He have a highly contrasted solubility in groundwater and this assumption is not always valid; for instance, in the KusatsuShirane volcanic area, Sano et al. (1994) observed a positive correlation between the temperature of the fumaroles and hot springs and the $\left(\mathrm{CO}_{2} /{ }^{3} \mathrm{He}\right)$ ratio: $\left(\mathrm{CO}_{2} /{ }^{3} \mathrm{He}\right)$ values varied by a factor 2 for a temperature 
range $23{ }^{\circ} \mathrm{C}-102{ }^{\circ} \mathrm{C}$, even though the $\delta^{13} \mathrm{C}$ was not affected, thus showing that elemental fractionation due to physico-chemical processes affecting $\mathrm{CO}_{2}$ may be important. In la Soufrière fumaroles however, the temperature range is much narrower $\left(94^{\circ} \mathrm{C}-108.5{ }^{\circ} \mathrm{C}\right)$, therefore such an effect is not expected to be seen. Nevertheless, in the Bouillante area in particular, the physico-chemical interaction between magmatic $\mathrm{CO}_{2}$ and the inorganic marine carbon system may decrease the $\left(\mathrm{CO}_{2} /{ }^{3} \mathrm{He}\right)$ ratio due to the partial transformation of magma-derived $\mathrm{CO}_{2}$ into marine bi-carbonate and carbonate, as observed for instance by Pedroni et al. (1999) for the gases of a Bouillante thermal spring (Table 1 ) with a $\left(\mathrm{CO}_{2} /{ }^{3} \mathrm{He}\right)$ value as low as $0.3 \times 10^{9}$ and a high helium content of $204 \mathrm{ppm}$. Taking account of this potential influence, the $\left(\mathrm{CO}_{2} /{ }^{3} \mathrm{He}\right)-\delta^{13} \mathrm{C}$ diagram suggests that the proportion of MORB-type upper mantle end-member in the Bouillante gases is about the same as in La Soufrière fumaroles. Therefore, the strong difference in the $\left({ }^{3} \mathrm{He} /{ }^{4} \mathrm{He}\right)$ ratio between both systems clearly points to an additional radiogenic ${ }^{4} \mathrm{He}$ component in the Bouillante geothermal reservoir.

This can be further investigated by plotting $\left({ }^{3} \mathrm{He} /{ }^{4} \mathrm{He}\right)$ ratios as a function of $\delta^{13} \mathrm{C}$ (Fig. 5), using the additional Eq. (4) for helium isotopes:

$\mathrm{R}\left({ }^{3} \mathrm{He} / \mathrm{CO}_{2}\right)=\mathrm{MR}_{\mathrm{M}}\left({ }^{3} \mathrm{He} / \mathrm{CO}_{2}\right)_{\mathrm{M}}+\mathrm{LR}_{\mathrm{L}}\left({ }^{3} \mathrm{He} / \mathrm{CO}_{2}\right)_{\mathrm{L}}+\mathrm{SR}_{\mathrm{S}}\left({ }^{3} \mathrm{He} / \mathrm{CO}_{2}\right)_{\mathrm{S}}$

where $R_{M}, R_{L}$, and $R_{S}$ are the ${ }^{3} \mathrm{He} /{ }^{4} \mathrm{He}$ ratios of the three end-members. $R_{M}$ value is taken equal to $8.25 R_{a}$, and $R_{L}$ and $R_{S}$ are $<0.05 R_{a}$. Using the $S$ value determined above ( $S=7 \%$ ), the calculated values of $\mathrm{M}$ and $\mathrm{L}$ for $\mathrm{La}$ Soufrière are $17 \%$ and $76 \%$ respectively, in excellent agreement with the values determined from the $\left(\mathrm{CO}_{2} /{ }^{3} \mathrm{He}\right)$ vs $\delta^{13} \mathrm{C}$ diagram. On the contrary, the ${ }^{3} \mathrm{He} /{ }^{4} \mathrm{He}$ ratio of the Bouillante system clearly departs from the mixing curve (Fig. 5), demonstrating that the lower ${ }^{3} \mathrm{He} /{ }^{4} \mathrm{He}$ ratio is due to the addition of extraneous radiogenic helium, presumably from the surrounding host rocks. Such a subsurface crustal contamination has been observed in many instances (see Gasparon et al., 1994 and references therein) and is also responsible for the decreasing trend in the $\left({ }^{3} \mathrm{He} /{ }^{4} \mathrm{He}\right)$ values of volcanic gases which is classically observed away from the main conduit of volcanic edifices (Sano et al., 1984, 1995). Fig. 6 shows that such a spatial trend also characterizes all measured fluids in Guadeloupe. It also shows that the low $\left({ }^{3} \mathrm{He} /{ }^{4} \mathrm{He}\right)$ values observed for the Bouillante hydrothermal system are consistent with this spatial trend. This strengthens our view, based on the $\left(\mathrm{CO}_{2} /{ }^{3} \mathrm{He}\right)$ vs $\delta^{13} \mathrm{C}$ and $\left({ }^{3} \mathrm{He} /{ }^{4} \mathrm{He}\right)$ vs $\delta^{13} \mathrm{C}$ relationships, that La Soufrière and Bouillante systems, despite their marked hydrogeological differences, are fed by the same magmatic source, and that the strong contrast in

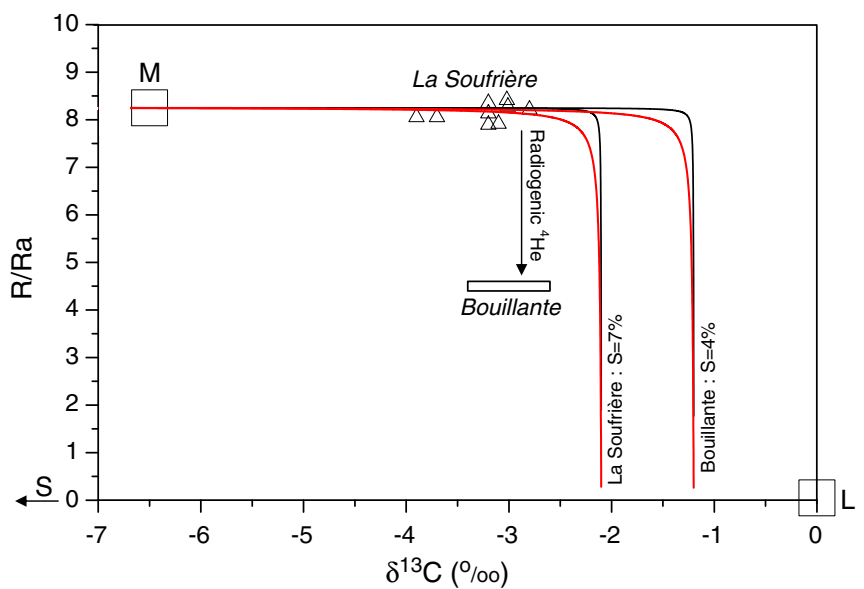

Fig. 5. $\left({ }^{3} \mathrm{He} /{ }^{4} \mathrm{He}\right)$ vs $\delta^{13} \mathrm{C}$ diagram for summit fumaroles and Bouillante gases in relation to the three major end-members contributing to $\mathrm{He}$ and $\mathrm{CO}_{2}$ in geothermal fluids ( $\mathrm{M}$ represents the upper mantle end-member, $\mathrm{L}$ and $\mathrm{O}$ represent the marine carbonate-limestone and organic material, respectively). The black rectangle represents the $\left({ }^{3} \mathrm{He} /{ }^{4} \mathrm{He}\right)-\delta^{13} \mathrm{C}$ domain for Bouillante (see Table 1).

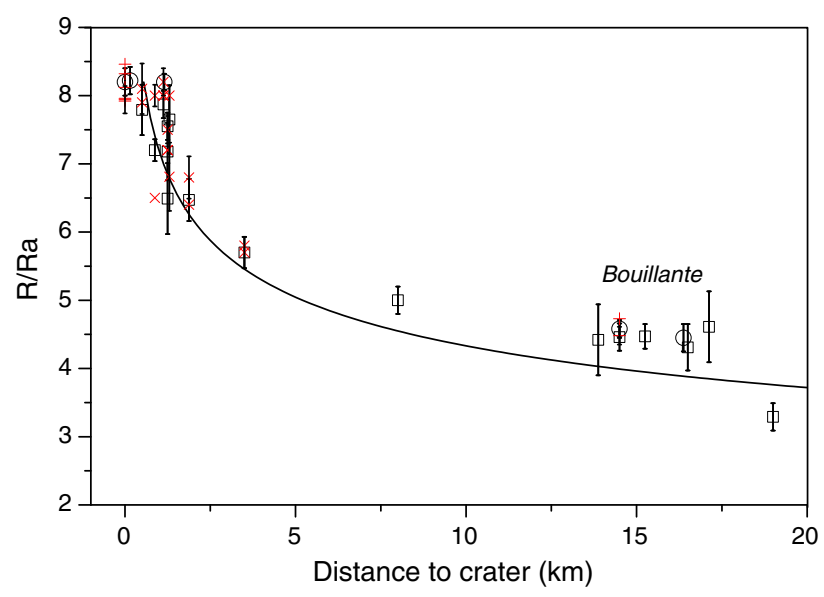

Fig. 6. Decreasing trend of the ${ }^{3} \mathrm{He} /{ }^{4} \mathrm{He}$ ratios with distance to the crater for gases (black circles) and waters (black squares). In red, literature data for gases $(+)$ by Van Soest et al., 1998; Pedroni et al., 1999; Ruzié et al., 2012 and for waters (x) (Ruzié et al., 2013).

the ${ }^{3} \mathrm{He} /{ }^{4} \mathrm{He}$ values between both systems has to be ascribed to radiogenic helium contamination by the surrounding wallrock.

\section{Conclusions}

A substantial number of samples were collected over the last thirty years from the volcanic and geothermal manifestations of Guadeloupe in order to study their helium and carbon isotope geochemistry. Based on this data set, our main conclusions are as follows:

${ }^{3} \mathrm{He} /{ }^{4} \mathrm{He}$ values in the fumarolic gases around La Soufrière volcano are quite homogeneous and have remained quite stable over the sampling period (1980-2012). The average value of $8.2 \pm 0.2 R_{a}$ indicates that the volcano is tapping a MORB-like source.

In contrast, the ${ }^{3} \mathrm{He} /{ }^{4} \mathrm{He}$ values for the Bouillante deep geothermal reservoir ( $\left.4.5 \pm 0.1 R_{a}\right)$ are strikingly lower.

$\mathrm{He}-\mathrm{C}$ relationships strongly suggest that both systems are fed by the same magmatic source, the marked difference in their ${ }^{3} \mathrm{He} /{ }^{4} \mathrm{He}$ being due to radiogenic ${ }^{4} \mathrm{He}$ accumulation from the host rock in the confined Bouillante deep aquifer.

This conclusion is strengthened by the spatial distribution of the ${ }^{3} \mathrm{He} /{ }^{4} \mathrm{He}$ ratio for all studied sites, which shows that La Soufrière volcano and the Bouillante geothermal system are the two end-members of a spatial decreasing trend for ${ }^{3} \mathrm{He} /{ }^{4} \mathrm{He}$ ratios with distance from $\mathrm{La}$ Soufrière dome, due to an increasing radiogenic ${ }^{4} \mathrm{He}$ contamination by the host rock away from the volcanic axis.

\section{References}

Abou Akar, A., Matray, J.M., Brach, M., 1992. Etude géochimique du fluide géothermal du puits B02 (centrale EDF) et des sources thermales de la région de Bouillante (Guadeloupe). Rapport BRGM R36203/IRG SGN 92.

Allard, P., 1983. The origin of hydrogen, carbon, sulfur, nitrogen and rare gases in volcanic exhalations: evidence from isotope geochemistry. In: Tazieff, H., Sabroux, J.C. (Eds.), Forecasting Volcanic Events. Elsevier, Amsterdam, pp. 337-386.

Allard, P., Delibrias, G., Dimon, B., Labeyrie, J., 1983. Implications of carbon and helium isotopes in volcanic gases from la Soufrière of Guadeloupe, Lesser Antilles. Abstract Presented at 1983 IAVCEI Symp. on Volcanic Gases, Hamburg, Germany, 43.

Allard, P. Jean-Baptiste, P. D'Alessandro, W., Parello, F, Parisi, B., Flehoc, C., 1997. Mantlederived helium and carbon in groundwaters and gases of Mount Etna, Italy. Earth Planet. Sci. Lett. 148 (501-516).

Ballentine, C.J., Burnard, P.G., 2002. Production, release and transport of noble gases in the continental crust. In: Porcelli, D., Ballentine, C.J., Wieler, R. (Eds.), Noble Gases in Geochemistry and Cosmochemistry. Rev. Mineral. Geochem, 47, pp. 481-538.

Ballentine, C.J., Burgess, R., Marty, B., 2002. Tracing fluid origin, transport and interaction in the crust. In: Porcelli, D., Ballentine, C.J., Wieler, R. (Eds.), Noble gases in Geochemistry and Cosmochemistry. Rev. Mineral. Geochem, 47, pp. 539-614.

Bigot, S., Boudon, G., Semet, M.P., Hammouya, G., 1994. Chemical tracing of groundwater circulation in Grande Découverte volcano (la Soufrière), Guadeloupe. CR Acad. Sci. Paris 318 (série II), 1215-1221. 
Boudon, G., Semet, M.P., Vincent, P.M., 1989. The evolution of la Grande Découverte (La Soufrière) volcano, Guadeloupe. In: Latter, J. (Ed.), Volcanic Hazards: Assessment and Monitoring. IAVCEI Proceedings in Volcanology, Berlin, pp. 86-109.

Boudon, G., Komorowski, J.C., Villemant, B., Semet, M.P., 2008. A new scenario for the last magmatic eruption of La Soufriere of Guadeloupe (Lesser Antilles) in 1530 A.D. Evidence from stratigraphy radiocarbon dating and magmatic evolution of erupted products. J. Volcanol. Geotherm. Res. 178, 474-490.

Briden, J.C., Rex, D.C., Fuller, A.M., Tomblin, J.F., 1979. K-Ar geochronology and paleomagnetism of volcanic rocks in the Lesser Antilles arc. Philos. Trans. R. Soc. Lond. 291, 211-228.

Brombach, T., Marini, L., Hunziker, J.C., 2000. Geochemistry of the thermal springs and fumaroles of Basse-Terre Island, Guadeloupe, Lesser Antilles. Bull. Volcanol. 61, 477-490.

Cormy, G., Demians D'Archambaud, J., Surcin, J., 1970. Prospection géothermique aux Antilles Françaises, Guadeloupe et Martinique. Geothermics 2, 57-72.

Demians D'Archambaud, J., Surcin, J., 1972. Recherches géothermiques en Guadeloupe. Rev. Géogr. Phys. Géol. Dyn. 14, 211-228.

Demians D'Archambaud, J., Surcin, J., 1976. Recherche d'énergie géothermique en Guadeloupe. Bull. BRGM 4, 365-373.

Fabriol, R., Ouzounian, G., 1985. Prospection géothermique des zones de Bouillante et de la Soufrière (Guadeloupe): modèle hydrogéochimique. Rapport BRGM 85 SGN 433 GTH.

Fischer, T.P., Marty, B., 2005. Volatile abundances in the sub-arc mantle: insights from volcanic and hydrothermal gas discharges. J. Volcanol. Geotherm. Res. 140, 205-216.

Gasparon, M., Hilton, D.R., Varne, R., 1994. Crustal contamination processes traced by helium isotopes: examples from the Sunda Arc. Indonesia. Earth Planet. Sci. Lett. $126,15-22$.

Graham, D.W., 2002. Noble gas isotope geochemistry of Mid-Ocean Ridge and Ocean Island Basalts: characterization of mantle source reservoirs. In: Porcelli, D., Ballentine, C.J., Wieler, R. (Eds.), Noble Gases in Geochemistry and Cosmochemistry. Rev. Mineral. Geochem, 47, pp. 247-317.

Hilton, D.R., 1996. The helium and carbon isotope systematics of a continental geothermal system: results from monitoring studies at Long Valley caldera (California, USA). Chem. Geol. 127, 269-295.

Hoefs, J., 1980. Stable Isotope Geochemistry. Springer, Berlin (208 pp.).

Javoy, M., Pineau, F., Delorme, H., 1986. Carbon and nitrogen isotopes in the mantle. Chem. Geol. 57, 41-62.

Jean-Baptiste, P., Mantisi, F., Dapoigny, A., Stievenard, M., 1992. Design and performance of a mass spectrometric facility for measuring helium isotopes in natural waters and for low-level tritium determination by the ${ }^{3} \mathrm{He}$ ingrowth method. Appl. Radiat. Isot. 43, 881-891.

Jean-Baptiste, P., Allard, P., Coutinho, R., Ferreira, T., Fourré, E., Queiroz, G., Gaspar, J.L., 2009. Helium isotopes in geothermal fluids of the Azores archipelago. Earth Planet. Sci. Lett. 281, 70-80.

Jean-Baptiste, P., Fourré, E., Dapoigny, A., Baumier, D., Baglan, N., Alanic, G., 2010. ${ }^{3} \mathrm{He}$ mass spectrometry for very low-level measurement of organic tritium in environmental samples. J. Environ. Radioact. 101, 185-190.

Kulongoski, J.T., Hilton, D.R., Barry, P.H., Esser, B.K., Hillegonds, D., Belitz, K., 2013. Volatile fluxes through the Big Bend section of the San Andreas Fault, California: helium and carbon-dioxide systematics. Chem. Geol. 339, 92-102.

MacDonald, R., Hawkesworth, C.J., Heath, E., 2000. The Lesser Antilles volcanic chain: a study in arc magmatism. Earth Sci. Rev. 49, 1-76.
Marty, B., Jambon, A., 1987. C $\beta^{3} \mathrm{He}$ in volatiles fluxes from the solid Earth: implication for carbon geodynamics. Earth Planet. Sci. Lett. 82, 16-26.

Marty, B., Tolstikhin, I.N., 1998. CO $_{2}$ fluxes from mid-ocean ridges, arcs and plumes. Chem. Geol. 145, 233-248.

O'Nions, R.K., Oxburgh, E.R., 1988. Helium, volatiles fluxes and the development of continental crust. Earth Planet. Sci. Lett. 90, 331-347.

Oppenheimer, C., Fischer, T.P., Scaillet, B., 2013. Volcanic degassing: processes and impact In: Turekian, K., Holland, H. (Eds.), 2nd edition. Treatise on Geochemistry, vol. 4. Elsevier, Amsterdam, pp. 111-179.

Pascaline, H., Benauges, S., Jérémie, J.J., Blavoux, B., 1982. Hydrochimie et circulation des eaux de sources chaudes du massif de la Soufrière, Guadeloupe. Bull. Mus. Natn. Hist. Nat. 4, 137-155.

Pedroni, A., Hammerschmidt, K., Friedrichsen, H., 1999. He, Ne, Ar, and C systematics of geothermal emanations in the Lesser Antilles Arc. Geochim. Cosmochim. Acta 63 515-532.

Ruzié, L., Moreira, M., Crispi, O., 2012. Noble gas isotopes in hydrothermal volcanic fluids of La Soufrière volcano, Guadeloupe, Lesser Antilles arc. Chem. Geol. 304-305 158-165.

Ruzié, L., Aubaud, C., Moreira, M., Agrinier, P., Dessert, C., Gréau, C., Crispi, O., 2013. Carbon and helium isotopes in thermal springs of La Soufrière volcano (Guadeloupe, Lesser Antilles): implications for volcanological monitoring. Chem. Geol. 359, 70-80.

Samper, A, Quidelleur, X, Lahitte, P. Mollex, D., 2007. Timing of effusive volcanism and collapse events within an oceanic island arc: Basse-Terre, Guadeloupe archipelago (Lesser Antilles). Earth Planet. Sci. Lett. 258, 175-191.

Sanjuan, B., Lasne, E., Brach, M., Vaute, L., 1999. Bouillante geothermal fleld (Guadeloupe): geochemical monitoring during the stimulation operation and critical evaluation of chemical and isotopic field data. BRGM Report, R40646, Orléans, France (99 pp).

Sano, Y., Marty, B., 1995. Origin of carbon in fumarolic gas from island arcs. Chem. Geol. $119,265-274$

Sano, Y., Nakamura, Y., Wakita, H., Urabe, A., Tominaga, T., $1984 .{ }^{3} \mathrm{He}$ emission related to volcanic activity. Science 224, 150-151.

Sano, Y., Hirabayashi, J., Ohba, T., Gamo, T., 1994. Carbon and helium isotopic ratios at Kusatsu-Shirane volcano, Japan. Appl. Geochem. 9, 371-377.

Sano, Y., Gamo, T., Notsu, K., Wakita, H., 1995. Secular variations of carbon and helium isotopes at Izu-Oshima volcano, Japan. J. Volcanol. Geotherm. Res. 64, 83-94.

Stuart, F.M., Lass-Evans, S., Fitton, J.G., Ellam, R.M., 2003. Hign ${ }^{3} \mathrm{He} /{ }^{4} \mathrm{He}$ ratios in picritic basalts from Baffin Island and role of a mixed reservoir in mantle plumes. Nature 424, 57-59.

Van Soest, M.C., Hilton, D.R., Kreulen, R., 1998. Tracing crustal and slab contributions to arc magmatism in the Lesser Antilles island arc using helium and carbon relationship in geothermal fluids. Geochim. Cosmochim. Acta 62, 3323-3335.

Villemant, B., Hammouya, G., Michel, A., Semet, M.P., Komorowski, J.-C., Boudon, G., Cheminée, J.-L., 2005. The memory of volcanic waters: shallow magma degassing revealed by halogen monitoring in thermal springs of La Soufrière volcano (Guadeloupe, Lesser Antilles). Earth Planet. Sci. Lett. 237, 710-728.

Weiss, R.F., 1971. Solubility of helium and neon in water and seawater. J. Chem. Eng. Data 16, 235-241. 